\title{
Pruritus Features in Children with End-Stage Renal Disease Underwent Dialysis: A Cross-Sectional Study
}

\author{
Mehryar Mehrkash, ${ }^{1}$ Seyed-javad Golestaneh, ${ }^{2}$ Yahya Madihi, ${ }^{1}$ Fatemeh Paknazar, ${ }^{3}$ \\ Mahdi Hadian, ${ }^{2}$ Mojtaba Akbari, ${ }^{4}$ and Bahareh Abtahi-Naeini ${ }^{5,6}$ \\ ${ }^{1}$ Imam Hossein Children's Hospital and Child Growth and Development Research Center, Research Institute for Primordial \\ Prevention of Non Communicable Disease, Isfahan University of Medical Sciences, Isfahan, Iran \\ ${ }^{2}$ School of Medicine, Isfahan University of Medical Sciences, Isfahan, Iran \\ ${ }^{3}$ Social Determinants of Health Research Center, Semnan University of Medical Sciences, Semnan, Iran \\ ${ }^{4}$ Department of Epidemiology, School of Health, Isfahan University of Medical Sciences, Isfahan, Iran \\ ${ }^{5}$ Pediatric Dermatology Division of Department of Pediatrics, Imam Hossein Children's Hospital, Isfahan University of \\ Medical Sciences, Isfahan, Iran \\ ${ }^{6}$ Skin Diseases and Leishmaniasis Research Center, Isfahan University of Medical Sciences, Isfahan, Iran
}

Correspondence should be addressed to Bahareh Abtahi-Naeini; abtahi.bahareh@yahoo.com

Received 13 March 2021; Revised 6 July 2021; Accepted 19 July 2021; Published 28 July 2021

Academic Editor: Lavjay Butani

Copyright (c) 2021 Mehryar Mehrkash et al. This is an open access article distributed under the Creative Commons Attribution License, which permits unrestricted use, distribution, and reproduction in any medium, provided the original work is properly cited.

\begin{abstract}
Objective. Evaluation of the pruritus features in children with end-stage renal disease (ESRD) who underwent dialysis at an academic tertiary pediatric dialysis center. Methods. This cross-sectional study was conducted at an academic tertiary pediatric dialysis center, Isfahan, Iran. The reviewed medical records of the children included their characteristics, dialysis properties, and laboratory parameters. The 4-item itch questionnaire was utilized to assess distribution, severity, frequency, and associated sleeping disorders. Results. Thirty ESRD patients with pruritus, including 23 males (76.7\%) with a mean age of $11.7 \pm 3.64$ years, were recruited. The most common cause of CKD was nephronophthisis (23.3\%). The median total score of pruritus was 5 (range: 3-15). The distribution score of pruritus was directly correlated with the age (Spearman's rho $=0.42, P=0.02$ ) and serum level of parathyroid hormone $(\mathrm{PTH})$ (Spearman's rho $=0.42, P=0.04)$. In the reduced multiple logistic regression model, the increasing level of serum calcium was associated with increased odds of having total pruritus score $\geq 5$ (OR (odds ratio): 4.5; $95 \%$ CI 1.12 to 18.05). In addition, an increase in age for one year was found to be associated with $50 \%$ higher odds of having total pruritus score $\geq 5$ (OR: 1.5; 95\% CI 1.03 to 2.18). Conclusion. Increased level of serum Ca and higher age were associated with increased odds of having more severe pruritus score in children.
\end{abstract}

\section{Introduction}

Chronic kidney disease (CKD) and end-stage renal disease (ESRD) and its complications are a growing problem worldwide in children $[1,2]$. Regardless of CKD cause, it may be accompanied with various skin lesions, including pruritus [3-5]. Pruritus, as a common and distressing symptom among patients with $\mathrm{CKD}$, poses a high burden, and decreases quality of patients' life $[6,7]$. Etiology of uremic pruritus may comprise several factors, including xerosis, peripheral neuropathy, mast cell hyperplasia, increased serum level of histamine, vitamin A, parathyroid hormone $(\mathrm{PTH})$, and certain inflammatory factors, such as interleukin-2 (IL2) $[8,9]$.

Finding an approach to the treatment of children with pruritus is believed to be a challenging issue for two major reasons; primarily, there are not many studies concerning itching and associated factors in children and adolescents 
with ESRD; secondly, the exact etiology and pathophysiology of CKD-associated pruritus remains unclear and is uncommon in children [10].

Knowledge about the characteristics and related factors to CKD-associated pruritus is important for the identification of possible risk factors, implementing effective and appropriate intervention for improving the clinical manifestation, and allocating enough health care resources.

Certain studies have been conducted to evaluate the clinical characteristics and etiology of uremic pruritus in adult patients [11-13]; however, there are a limited number of studies with epidemiological information on ESRDassociated pruritus in children $[3,14]$. The scarcity of epidemiological information is even more pronounced in developing countries. Thus, it is necessary to conduct further studies on the role of clinicoepidemiogical and socioeconomic factors in the management of uremic pruritus. Therefore, the current study is aimed at providing itching characteristics and exploring its association with the selected related factors in ESRD-associated pruritus in children under dialysis.

\section{Material and Methods}

2.1. Study Population. This cross-sectional study was conducted at an academic tertiary pediatric dialysis center in Imam Hossein Children's Hospital, affiliated to Isfahan University of Medical Sciences. The project was approved by the Ethical Committee of Isfahan University of Medical Sciences (IR.MUI.MED.REC.1399.571), in agreement with the last version of Helsinki Declaration. Written informed consent for participation was obtained from all the children and their parents/guardian after the objective, and the procedure of our work were completely explained.

The inclusion criteria for case selection were (i) definite diagnosis of ESRD, (ii) initiation of dialysis at least 6 months beforehand, (iii) age at the onset of CKD below 18 years, and (iv) children with 3-18 years old. The exclusion criteria were changes in the mental status making the patient unable to make a detailed assessment of itch, known case of severe liver disease, and history of chronic dermatologic diseases, such as atopic dermatitis, psoriasis, and scabies. Additionally, those who refused to participate were excluded.

2.2. Data Collection. The reviewed medical records of the children included their sex, age, causes of ESRD, dialysis type and duration, complications related to dialysis, age at onset of dialysis, urinary output status, comorbidity, and history of consanguineous marriage of parents. The laboratory tests were performed according to a protocol of routine blood tests for the dialyzed patients and were measured with routine methods in our center. Serum blood urea nitrogen (BUN), creatinine $(\mathrm{Cr})$, calcium $(\mathrm{Ca})$, phosphorus $(\mathrm{P})$, $\mathrm{Ca} \times \mathrm{P}$ product, and parathyroid hormone (PTH) level were determined in all the participants. These parameters were assessed over the previous 6 months and the mean, minimum, and maximum of each parameter were noted.

2.3. Pruritus Assessment. The 4-item itch questionnaire (4IIQ) was utilized to assess distribution, severity, frequency, and the associated sleeping disorders [1]. The questionnaire estimated the extent of pruritus (1-3 points), intensity (1-5 points), frequency (1-5 points), and sleep disturbances (0-6 points) caused by itching during the seven days prior to the examination. The ratings ranged from 3 (mild pruritus) to 19 points (very severe itching) [2, 3].

2.4. Statistical Analysis. Numerical variables were described with the mean, standard deviation (SD), median, and range (minimum and maximum) of the values. Frequency distribution tables were used to report the count and percentage of categorical variables. Based on the total pruritus score of 5 , the patients were divided into two groups with lower $(\leq 5)$ and higher $(>5)$ severity. The analysis was performed through two stages, namely, the preliminary stage to select the most important variables, and the main stage for modeling the selected variables. The marginal relationship between each categorical variable and the two groups was initially evaluated via Chi-square or Fisher's exact tests; the rest of the variables (numerical ones) were compared between the two groups employing $t$-test or Mann-Whitney $U$ test regarding the normal distribution assumption. The normal distribution assumption was checked with the Shapiro-Wilk test. The associations between the numerical variables and pruritus scores were also assessed with Spearman's rho correlation coefficients. The variables that were significantly $(P<0.05)$ or liberally $(P<0.2)$ related to the pruritus score in the first stage entered the final analysis. In this stage, to examine the relationship between each of the explanatory variables and being in the group with a higher score as the dependent variable, simple and multiple logistic regression models were fitted on the data and the final reduced model was obtained via stepwise (backward LR) method and was interpreted. Statistical analysis was performed with SPSS-18 software at $95 \%$ confidence level for the statistical tests.

\section{Results}

3.1. Demographic and Laboratory Data/Study Population. Thirty CKD children with pruritus were recruited. The mean age of the patients was $11.7 \pm 3.64$ (range 3 -18) years, and there were 23 males $(76.7 \%)$. The most frequent causes of CKD were nephronophthisis (23.3\%), hypoplastic kidney (10\%), and FSGS (10\%). MBD, anemia, and HTN were noted in $93.3 \%, 90 \%$, and $80 \%$ of the patients, respectively. The mean dialysis duration was $34 \pm 23.4$ (range 9-108) months. Table 1 represents the demographics and laboratory parameters of the patients enrolled in this study.

3.2. Pruritus Features. The mean total score of pruritus was $5.37 \pm 2.41$. The median total score was 5 (range: $3-15$ ). Seventeen patients $(56.7 \%)$ had total score $\geq 5$. Frequency of pruritus features included distribution, severity, episodes, and sleep disturbances in the patients, which are shown in Table 2. Dry skin was observed in all the subjects. Most patients had multiple location distributions (18/30), itching with the need to scratch, but without excoriation (14/30), four short episodes or one long episode (29/30), and no episode of awakening due to pruritus (27/30) (Table 2). 
TABle 1: Clinical characteristics and laboratory data of chronic kidney disease (CKD) children with pruritus.

\begin{tabular}{|c|c|c|}
\hline \multicolumn{3}{|l|}{ Sex } \\
\hline Male $(n, \%)$ & 23 & 76.7 \\
\hline Female $(n, \%)$ & 7 & 23.3 \\
\hline \multicolumn{3}{|l|}{ Dialysis type } \\
\hline Peritoneal $(n, \%)$ & 11 & 36.7 \\
\hline Hemodialysis $(n, \%)$ & 19 & 63.3 \\
\hline \multicolumn{3}{|l|}{ Complication of dialysis } \\
\hline Anemia $(n, \%)$ & 27 & 90.0 \\
\hline $\operatorname{MBD}(n, \%)$ & 28 & 93.3 \\
\hline $\operatorname{HTN}(n, \%)$ & 24 & 80.0 \\
\hline Acidosis $(n, \%)$ & 18 & 60.0 \\
\hline Lipid disorder $(n, \%)$ & 5 & 16.7 \\
\hline Thyroid disease $(n, \%)$ & 4 & 13.3 \\
\hline \multicolumn{3}{|l|}{ Urinary output } \\
\hline Complete anuria $(n, \%)$ & 7 & 23.3 \\
\hline Relative anuria $(n, \%)$ & 13 & 43.3 \\
\hline Normal $(n, \%)$ & 6 & 20.0 \\
\hline Polyuria $(n, \%)$ & 4 & 13.3 \\
\hline \multicolumn{3}{|l|}{ Comorbidity } \\
\hline No $(n, \%)$ & 21 & 70.0 \\
\hline Yes $(n, \%)$ & 9 & 30.0 \\
\hline \multicolumn{3}{|l|}{ Consanguineous marriage } \\
\hline Yes $(n, \%)$ & 23 & 76.7 \\
\hline No $(n, \%)$ & 7 & 23.3 \\
\hline \multicolumn{3}{|l|}{ Age (year) } \\
\hline Mean (SD) & 11.7 & $(3.64)$ \\
\hline Median (min-max) & 13 & $3-18$ \\
\hline \multicolumn{3}{|l|}{ Dialysis duration (month) } \\
\hline Mean (SD) & 34 & $(23.4)$ \\
\hline Median (min-max) & 24 & $9-108$ \\
\hline \multicolumn{3}{|l|}{ Age at onset of dialysis (year) } \\
\hline Mean (SD) & 9.09 & $(4.15)$ \\
\hline Median (min-max) & 8.5 & $0-15$ \\
\hline \multicolumn{3}{|l|}{ Serum BUN (mg/dl) } \\
\hline Mean (SD) & 53.27 & $(24.19)$ \\
\hline Median (min-max) & 49.5 & $24-124$ \\
\hline \multicolumn{3}{|l|}{ Serum Cr (mg/dl) } \\
\hline Mean (SD) & 6.82 & $(2.57)$ \\
\hline Median (min-max) & 6.6 & $1.35-11.3$ \\
\hline \multicolumn{3}{|l|}{ Serum Ca $(\mathrm{mg} / \mathrm{dl})$} \\
\hline Mean (SD) & 9.13 & $(0.92)$ \\
\hline Median (min-max) & 9.2 & $7.2-11.4$ \\
\hline \multicolumn{3}{|l|}{ Serum P (mg/dl) } \\
\hline Mean (SD) & 5.43 & $(1.49)$ \\
\hline Median (min-max) & 5.4 & $2.7-9.2$ \\
\hline \multicolumn{3}{|l|}{ Serum $\mathrm{Ca} \times \mathrm{P}$} \\
\hline Mean (SD) & 49.7 & $(14.46)$ \\
\hline Median (min-max) & 47.7 & $26.2-75.4$ \\
\hline \multicolumn{3}{|l|}{ Serum PTH (ng/ml) } \\
\hline Mean (SD) & 524.12 & $(313.53)$ \\
\hline Median (min-max) & 594 & $92-1079$ \\
\hline
\end{tabular}

MBD: mineral bone disorder; HTN: hypertension; BUN: blood urea nitrogen; Cr: creatinine; Ca: calcium; P: phosphorus; PTH: parathyroid hormone.
3.3. Characteristics of the Patients according to Total Pruritus Score. Tables 3 and 4 depict the distribution of general characteristics as well as the laboratory parameters and continuous variables according to the total pruritus score. Distribution of sex, dialysis type, dialysis-related complications, urinary outputs, and consanguineous marriage of parents were not significantly different between total pruritus score $<5$ and $\geq 5(P>0.05)$ (Table 3$)$. The subjects with higher serum level of $\mathrm{Ca}$ had higher total pruritus score $\geq 5$ $(9.41 \pm 0.83$ in total pruritus score $\geq 5$ versus $8.76 \pm 0.92$ in total pruritus score $<5 ; P=0.05$ ) (Table 4 ).

3.4. Correlation between Quantitative Variable and Pruritus Score. Correlation analysis was performed between the quantitative characteristic and pruritus score (Table 5). The distribution score of pruritus was directly correlated with the age of the patients (Spearman's rho $=0.42, P=0.02$ ) and the serum level of PTH (Spearman's rho $=0.42, P=0.04$ ). There were no significant correlations between the other laboratory parameters and pruritus score $(P>0.05$ for all correlations) (Table 5).

3.5. Logistic Regression Model for Association between Pruritus and Explanatory Variables. Table 6 demonstrates the results of simple, multiple, and reduced multiple logistic regression models concerning the association between total pruritus score and explanatory variables. In the reduced multiple logistic regression model, the increase in the level of serum Ca was associated with the increased odds of having total pruritus score $\geq 5$ (OR (odds ratio): 4.5; 95\% CI 1.12 to $18.05)$. In addition, an increase in age by one year was associated with $50 \%$ higher odds of having total pruritus score $\geq$ 5 (OR: 1.5; 95\% CI 1.03 to 2.18) (Table 6).

\section{Discussion}

Our study revealed that most patients had mild severity of pruritus. The results demonstrated that the increase in the level of serum $\mathrm{Ca}$ and patients' age were associated with increased odds of having more severe pruritus. These findings could provide new insights into the clinical and epidemiological aspects of pruritus associated with ESRD in children.

In line with the study by Schwab et al., most of the children in our study had mild pruritus severity [10].

In this paper, the increase in age was found to be related to the increased severity of pruritus. This relation between age and severity could be attributed to the CD4 T cell differentiation into the Th1 cell along with the increase in age. Increased Th1 will produce greater IL-2, which is an important mediator in uremic itching [10].

In our study, all the patients had dry skin, possibly due to disturbed skin barrier that is intensified as the disease progresses and when the CKD progresses to ESRD; it seems logical in the presence of pruritus in most patients $[3,15]$. Moreover, none of our children used any kinds of moisturizers during the two months before. Meanwhile, study in adults demonstrated that in patients undergoing hemodialysis, the use of emollients significantly improved dry skin [16]. 
TABLE 2: Pruritus features of patients enrolled in the study.

\begin{tabular}{|c|c|c|c|}
\hline Pruritus feature & Categories & Count & $\%$ \\
\hline \multirow{3}{*}{ Distribution } & Single location & 11 & 36.7 \\
\hline & Multiple locations & 18 & 60.0 \\
\hline & Generalized & 1 & 3.3 \\
\hline \multirow{5}{*}{ Severity } & Without the need to scratch & 6 & 20.0 \\
\hline & With the need to scratch but without excoriation & 14 & 46.7 \\
\hline & Unrelieved by scratching but without excoriation & 5 & 16.7 \\
\hline & Accompanied by excoriation & 4 & 13.3 \\
\hline & Totally restless & 1 & 3.3 \\
\hline \multirow{2}{*}{ Frequency } & Four short episodes or one long episode & 29 & 96.7 \\
\hline & Continuous & 1 & 3.3 \\
\hline \multirow{2}{*}{ Sleep disturbances } & One episode of awakening due to pruritus & 2 & 6.7 \\
\hline & Two episodes of awakening due to pruritus & 1 & 3.3 \\
\hline
\end{tabular}

TABLE 3: Distribution of general characteristics of chronic kidney disease (CKD) children with pruritus according to the total pruritus score.

\begin{tabular}{|c|c|c|c|c|c|c|}
\hline \multirow{3}{*}{ Characteristics } & & \multicolumn{4}{|c|}{ Pruritus } & \multirow{3}{*}{$P$ value } \\
\hline & & \multicolumn{2}{|c|}{ Total score $<5$} & \multicolumn{2}{|c|}{ Total score $\geq 5$} & \\
\hline & & Count & $\%$ & Count & $\%$ & \\
\hline \multirow{2}{*}{ Sex } & Male & 11 & 84.6 & 12 & 70.6 & \multirow{2}{*}{0.43} \\
\hline & Female & 2 & 15.4 & 5 & 29.4 & \\
\hline \multirow{2}{*}{ Dialysis type } & Peritoneal & 5 & 38.5 & 6 & 35.3 & \multirow{2}{*}{0.99} \\
\hline & Hemodialysis & 8 & 61.5 & 11 & 64.7 & \\
\hline \multirow{2}{*}{ Anemia } & No & 0 & 0 & 3 & 17.6 & \multirow{2}{*}{0.24} \\
\hline & Yes & 13 & 100.0 & 14 & 82.4 & \\
\hline \multirow{2}{*}{$\mathrm{MBD}$} & No & 0 & 0 & 2 & 11.8 & \multirow{2}{*}{0.49} \\
\hline & Yes & 13 & 100.0 & 15 & 88.2 & \\
\hline \multirow{2}{*}{ HTN } & No & 1 & 7.7 & 5 & 29.4 & \multirow{2}{*}{0.19} \\
\hline & Yes & 12 & 92.3 & 12 & 70.6 & \\
\hline \multirow{2}{*}{ Acidosis } & No & 4 & 30.8 & 8 & 47.1 & \multirow{2}{*}{0.46} \\
\hline & Yes & 9 & 69.2 & 9 & 52.9 & \\
\hline \multirow{2}{*}{ Lipid disorder } & No & 11 & 84.6 & 14 & 82.4 & \multirow{2}{*}{0.99} \\
\hline & Yes & 2 & 15.4 & 3 & 17.6 & \\
\hline \multirow{2}{*}{ Thyroid disease } & No & 11 & 84.6 & 15 & 88.2 & \multirow{2}{*}{0.99} \\
\hline & Yes & 2 & 15.4 & 2 & 11.8 & \\
\hline \multirow{2}{*}{ Comorbidity } & No & 8 & 61.5 & 13 & 76.5 & \multirow{2}{*}{0.44} \\
\hline & Yes & 5 & 38.5 & 4 & 23.5 & \\
\hline \multirow{2}{*}{ Urinary output } & Abnormal & 10 & 76.9 & 14 & 82.4 & \multirow{2}{*}{0.99} \\
\hline & Normal & 3 & 23.1 & 3 & 17.6 & \\
\hline \multirow{2}{*}{ Consanguineous marriage of parents } & No & 3 & 23.1 & 4 & 23.5 & \multirow{2}{*}{0.99} \\
\hline & Yes & 10 & 76.9 & 13 & 76.5 & \\
\hline
\end{tabular}

MBD: mineral bone disorder; HTN: hypertension.

Several studies have suggested that skin dryness in CKD is an important factor for pruritus associated with CKD $[17,18]$. Children with ESRD undergoing dialysis reported skin dryness more often than those receiving conservative treatment [3]. Disturbed skin barrier in skin dryness may either facilitate or aggravate the development or intensity of pruritus. The high prevalence of dry skin in our study may be associated with the selection criteria in which all the patients had ESRD compared to studies with multistage CKD.
In the present research, there were no significant differences between the type of hemodialysis and severity of itching. On the one hand, children undergoing peritoneal dialysis had less skin moisture than those undergoing hemodialysis and healthy controls and there was a significant correlation between lower moisturizing of the stratum corneum and itching [19]. On the other hand, skin dryness may be influenced by race, accompanying illnesses, and various environmental factors [20]. Therefore, it seems as though the 
TABLE 4: Laboratory parameters and continuous variables chronic kidney disease (CKD) children with pruritus according to the total pruritus score.

\begin{tabular}{|c|c|c|c|c|c|c|c|c|c|c|c|c|}
\hline \multirow{3}{*}{\multicolumn{2}{|c|}{ Variables }} & \multicolumn{10}{|c|}{ Pruritus } & \multirow{3}{*}{$P$ value } \\
\hline & & \multicolumn{5}{|c|}{ Total score $<5$} & \multicolumn{5}{|c|}{ Total score $\geq 5$} & \\
\hline & & Mean & SD & Med & Min & Max & Mean & SD & Med & Min & Max & \\
\hline Age (year) & & 11 & 3.67 & 10 & 3 & 16 & 12.24 & 3.63 & 13 & 4 & 18 & 0.36 \\
\hline Duration of dialysis ( & ionths) & 32.77 & 26.87 & 24 & 9 & 108 & 34.94 & 21.24 & 36 & 12 & 96 & 0.51 \\
\hline Age of onset of dialys & (year) & 8.55 & 4.24 & 8 & 0 & 14 & 9.51 & 4.16 & 10 & 0 & 15 & 0.54 \\
\hline \multirow{3}{*}{ Serum BUN (mg/dl) } & Mean & 54.85 & 24.38 & 52 & 24 & 114 & 52.06 & 24.73 & 46 & 24 & 124 & 0.76 \\
\hline & Min & 43.15 & 17.84 & 41 & 15 & 82 & 37.29 & 18.94 & 32 & 16 & 92 & 0.39 \\
\hline & Max & 70.62 & 34.02 & 63 & 32 & 154 & 67 & 32.75 & 63 & 25 & 148 & 0.77 \\
\hline \multirow{3}{*}{ Serum Cr (mg/dl) } & Mean & 6.68 & 2.51 & 6.20 & 2.40 & 11.30 & 6.93 & 2.68 & 6.80 & 1.35 & 10.60 & 0.79 \\
\hline & Min & 5.80 & 2.32 & 5.90 & 2.00 & 11.00 & 5.85 & 2.50 & 5.70 & 1.30 & 10.20 & 0.96 \\
\hline & Max & 7.90 & 2.96 & 7.90 & 3.30 & 13.30 & 8.06 & 3.04 & 8.50 & 1.40 & 11.90 & 0.89 \\
\hline \multirow{3}{*}{ Serum Ca (mg/dl) } & Mean & 8.76 & 0.92 & 8.80 & 7.20 & 10.10 & 9.41 & 0.83 & 9.30 & 8.20 & 11.40 & 0.05 \\
\hline & Min & 7.82 & 1.11 & 8.30 & 6.20 & 9.40 & 8.71 & 1.05 & 8.80 & 7.20 & 10.80 & 0.03 \\
\hline & Max & 9.70 & 1.22 & 9.60 & 7.50 & 12.10 & 10.09 & 0.80 & 9.70 & 9.30 & 12.00 & 0.30 \\
\hline \multirow{3}{*}{ Serum P (mg/dl) } & Mean & 5.80 & 1.97 & 5.50 & 2.70 & 9.20 & 5.14 & 0.94 & 5.10 & 3.70 & 7.10 & 0.23 \\
\hline & Min & 4.68 & 0.96 & 4.70 & 3.20 & 7.30 & 4.54 & 0.65 & 4.70 & 3.20 & 5.50 & 0.97 \\
\hline & Max & 7.22 & 2.29 & 7.80 & 4.10 & 11.80 & 6.20 & 1.37 & 5.90 & 4.60 & 10.00 & 0.14 \\
\hline \multirow{3}{*}{ Serum PTH (ng/ml) } & Mean & 475.8 & 327.13 & 368 & 92 & 1079 & 562.07 & 309.26 & 660 & 140 & 1077 & 0.51 \\
\hline & Min & 374.91 & 322.6 & 178 & 92 & 1030 & 498.9 & 294.05 & 536 & 122 & 1029 & 0.33 \\
\hline & Max & 576.82 & 369.8 & 594 & 92 & 1128 & 625.3 & 340.4 & 710 & 140 & 1125 & 0.74 \\
\hline Serum $\mathrm{Ca} \times \mathrm{P}$ & & 50.87 & 17.93 & 47.85 & 26.19 & 75.44 & 48.80 & 11.66 & 47.50 & 30.34 & 68.40 & 0.71 \\
\hline
\end{tabular}

BUN: blood urea nitrogen; Cr: creatinine; Ca: calcium; P: phosphorus; PTH: parathyroid hormone; Med: median; Min: minimum; Max: maximum.

TABLE 5: Correlation between quantitative variables and pruritus score in terms of Spearman's rho.

\begin{tabular}{|c|c|c|c|c|c|c|}
\hline \multirow{2}{*}{\multicolumn{2}{|c|}{ Variable }} & \multicolumn{5}{|c|}{ Correlation coefficient ( $P$ value $)$} \\
\hline & & Distribution score & Severity score & Frequency score & Sleep score & Total score \\
\hline \multicolumn{2}{|l|}{ Age (year) } & $0.42(0.02)$ & $-0.12(0.54)$ & $0.01(0.96)$ & $0.02(0.89)$ & $0.17(0.37)$ \\
\hline \multicolumn{2}{|c|}{ Duration of dialysis (months) } & $0.21(0.27)$ & $-0.10(0.61)$ & $0.08(0.69)$ & $0.14(0.47)$ & $0.03(0.87)$ \\
\hline \multicolumn{2}{|c|}{ Age of onset of dialysis (year) } & $0.28(0.13)$ & $-0.13(0.50)$ & $0.05(0.78)$ & $0.04(0.83)$ & $0.09(0.64)$ \\
\hline \multirow{3}{*}{ Serum BUN (mg/dl) } & Mean & $0.08(0.68)$ & $0.13(0.47)$ & $0.22(0.23)$ & $0.06(0.74)$ & $0.03(0.84)$ \\
\hline & Min & $-0.06(0.76)$ & $0.07(0.70)$ & $0.17(0.36)$ & $0.12(0.50)$ & $-0.05(0.79)$ \\
\hline & Max & $0.13(0.47)$ & $0.13(0.48)$ & $0.19(0.31)$ & $0.01(0.92)$ & $0.04(0.82)$ \\
\hline \multirow{3}{*}{ Serum Cr (mg/dl) } & Mean & $0.12(0.51)$ & $-0.11(0.57)$ & $0.01(0.95)$ & $0.08(0.65)$ & $0.02(0.91)$ \\
\hline & Min & $0.11(0.57)$ & $-0.15(0.42)$ & $-0.01(0.95)$ & $0.05(0.77)$ & $-0.01(0.94)$ \\
\hline & Max & $0.10(0.60)$ & $-0.09(0.61)$ & $-0.05(0.78)$ & $0.09(0.64)$ & $0.02(0.90)$ \\
\hline \multirow{3}{*}{ Serum Ca $(\mathrm{mg} / \mathrm{dl})$} & Mean & $0.15(0.41)$ & $0.18(0.33)$ & $-0.19(0.31)$ & $0.09(0.62)$ & $0.29(0.12)$ \\
\hline & Min & $0.09(0.63)$ & $0.27(0.15)$ & $-0.14(0.46)$ & $0.06(0.76)$ & $0.34(0.06)$ \\
\hline & Max & $0.24(0.19)$ & $0.03(0.87)$ & $-0.19(0.30)$ & $0.11(0.57)$ & $0.17(0.36)$ \\
\hline \multirow{3}{*}{ Serum P (mg/dl) } & Mean & $-0.05(0.78)$ & $-0.23(0.22)$ & $-0.25(0.18)$ & $-0.13(0.51)$ & $-0.23(0.22)$ \\
\hline & Min & $0.23(0.23)$ & $-0.15(0.44)$ & $0.02(0.90)$ & $0.21(0.26)$ & $0.01(0.94)$ \\
\hline & Max & $-0.01(0.97)$ & $-0.23(0.21)$ & $-0.15(0.43)$ & $-0.12(0.52)$ & $-0.24(0.19)$ \\
\hline \multirow{3}{*}{ Serum PTH (ng/ml) } & Mean & $0.33(0.11)$ & $-0.13(0.54)$ & $0.23(0.28)$ & $0.05(0.80)$ & $0.06(0.79)$ \\
\hline & Min & $0.42(0.04)$ & $-0.12(0.58)$ & $0.20(0.34)$ & $0.06(0.76)$ & $0.12(0.56)$ \\
\hline & Max & $0.14(0.49)$ & $-0.12(0.57)$ & $0.14(0.50)$ & $-0.07(0.74)$ & $-0.05(0.80)$ \\
\hline Serum $\mathrm{Ca} \times \mathrm{P}$ & & $0.04(0.82)$ & $-0.17(0.36)$ & $-0.22(0.23)$ & $-0.09(0.64)$ & $-0.11(0.55)$ \\
\hline
\end{tabular}

BUN: blood urea nitrogen; Cr: creatinine; Ca: calcium; P: phosphorus; PTH: parathyroid hormone; Min: minimum; Max: maximum. 
TABLE 6: Relationship of each of the most important study variables with the pruritus score based on logistic regression models.

\begin{tabular}{|c|c|c|c|c|c|c|c|c|c|c|c|c|}
\hline \multirow{2}{*}{ Variables } & \multirow{2}{*}{$P$ value } & \multirow{2}{*}{$\mathrm{OR}^{1}$} & \multicolumn{2}{|c|}{$95 \% \mathrm{CI}$} & \multirow{2}{*}{$P$ value } & \multirow{2}{*}{$\mathrm{OR}^{2}$} & \multicolumn{2}{|c|}{$95 \% \mathrm{CI}$} & \multirow{2}{*}{$P$ value } & \multirow{2}{*}{$\mathrm{OR}^{3}$} & \multicolumn{2}{|c|}{$95 \% \mathrm{CI}$} \\
\hline & & & Lower & Upper & & & Lower & Upper & & & Lower & Upper \\
\hline Serum Ca (mean) & 0.06 & 2.6 & 0.93 & 7.21 & 0.55 & 2.3 & 0.15 & 34.09 & 0.03 & 4.5 & 1.12 & 18.05 \\
\hline Serum Ca (min) & 0.04 & 2.2 & 1.02 & 4.93 & 0.55 & 1.9 & 0.23 & 15.31 & - & - & - & - \\
\hline Serum P (max) & 0.15 & 0.73 & 0.47 & 1.12 & 0.41 & 0.81 & 0.49 & 1.35 & - & - & - & - \\
\hline HTN & 0.17 & 0.20 & 0.02 & 1.98 & 0.09 & 0.05 & 0.001 & 1.66 & 0.06 & 0.04 & 0.001 & 1.25 \\
\hline Age (year) & 0.36 & 1.1 & 0.89 & 1.36 & 0.04 & 1.5 & 1.02 & 2.20 & 0.03 & 1.5 & 1.03 & 2.18 \\
\hline
\end{tabular}

Ca: calcium; P: phosphorus; HTN: hypertension. ${ }^{1}$ Simple logistic regression models. ${ }^{2}$ Multiple logistic regression. model. ${ }^{3}$ Reduced multiple logistic regression model.

relation between the type of dialysis and the related factors with severity of pruritus is of great importance and should be addressed in the future.

The exact role of divalent ions, as the cause of or as a related factor to severity uremic pruritus, has been a controversial issue. Herein, the mean serum level of Ca during the previous 6 months was associated with intensity of pruritus. The effect of divalent ions on pruritus in children with ESRD is attributed to the nerve modulation and histamine release by mast cells [21]. Nonetheless, the role of histamine release by mast cells in ESRD-associated pruritus is uncertain. Thus, the effect of divalent ions, nerve modulation, and histamine release by mast cells, as a possible mechanism for uremic pruritus, still needs further investigation [22, 23].

Disappearance of uremic pruritus following parathyroidectomy was observed herein although this was a temporary event [10]. This finding is consistent with that of Harlim, reporting no significant correlations between the increased PTH and uremic pruritus [24]. Similarly, in our study, the increase in the PTH level was not correlated with the intensity of pruritus yet was interestingly associated with distribution of pruritus.

Our work had certain limitations, including the small sample size. Although the number of children with pruritus associated with ESRD was not large compared to the adult, it seemed to be an important factor for spotting significant differences between pruritus and the related factors. Lack of laboratory measurement exactly at the time of evaluation for pruritus was another limitation, which is also present in other similar studies. Furthermore, lack of some factors that were not evaluated in our study, such as plasma histamine level, cutaneous mast cell proliferation, opioid peptides, aluminum level, and inflammatory markers, could be considered another limitation; this might be important in children due to the problem in evaluation facilities.

Despite these limitations, our study investigated detailed characteristics of children with ESRD and applied a comprehensive scale to characterize itching in them. The present work could also provide references for the characteristics of pruritus in these children, about which there is limited data.

\section{Conclusion}

The obtained results herein suggested that pruritus associated with ESRD had a low score of severity among most children. Furthermore, the increase in the level of serum Ca and higher age could be associated with increased odds of having more severe pruritus in children. In the future, large-scale studies should be designed on pediatric populations to improve our understanding of this spectrum of disorders in children.

\section{Data Availability}

Data available on request.

\section{Conflicts of Interest}

The authors declare that there is no conflict of interests regarding the publication of this paper.

\section{References}

[1] D. Haffner, "Strategies for optimizing growth in children with chronic kidney disease," Frontiers in pediatrics, vol. 8, 2020.

[2] M. Moxey-Mims, "Kidney disease in African American children: biological and nonbiological disparities," American journal of kidney diseases: the official journal of the National Kidney Foundation, vol. 72, no. 5, pp. S17-s21, 2018.

[3] E. Wojtowicz-Prus, K. Kilis-Pstrusinska, A. Reich et al., "Disturbed skin barrier in children with chronic kidney disease," Pediatric Nephrology, vol. 30, no. 2, pp. 333-338, 2015.

[4] A. U. Solarin, P. Nourse, and P. Gajjar, "Vitamin D status of children with moderate to severe chronic kidney disease at a tertiary pediatric Center in Cape Town," Saudi journal of kidney diseases and transplantation: an official publication of the Saudi Center for Organ Transplantation, Saudi Arabia, vol. 30, no. 4, pp. 781-794, 2019.

[5] P. Dyachenko, A. Shustak, and D. Rozenman, "Hemodialysisrelated pruritus and associated cutaneous manifestations," International journal of dermatology, vol. 45, no. 6, pp. 664667, 2006.

[6] J. C. Szepietowski, A. Reich, and R. A. Schwartz, "Uraemic xerosis," Nephrology, Dialysis, Transplantation, vol. 19, no. 11, pp. 2709-2712, 2004.

[7] K. S. Ruidiaz-Gómez and L. F. Higuita-Gutiérrez, "Impact of chronic kidney disease on health-related quality of life in the pediatric population: meta-analysis," Jornal de pediatria, 2020.

[8] S. S. Swarna, K. Aziz, T. Zubair, N. Qadir, and M. Khan, "Pruritus associated with chronic kidney disease: a comprehensive literature review," Cureus, vol. 11, no. 7, 2019.

[9] C. G. Bolanos, N. M. Pham, R. D. Mair, T. W. Meyer, and T. L. Sirich, "Metabolomic analysis of uremic pruritus in patients on hemodialysis," PLoS One, vol. 16, no. 2, p. e0246765, 2021. 
[10] T. Mettang, C. Pauli-Magnus, and D. M. Alscher, "Uraemic pruritus-new perspectives and insights from recent trials," Nephrology Dialysis Transplantation, vol. 17, no. 9, pp. 15581563, 2002.

[11] X. Hu, Y. Sang, M. Yang, X. Chen, and W. Tang, "Prevalence of chronic kidney disease-associated pruritus among adult dialysis patients: a meta-analysis of cross-sectional studies," Medicine, vol. 97, no. 21, 2018.

[12] I. Narita, B. Alchi, K. Omori et al., "Etiology and prognostic significance of severe uremic pruritus in chronic hemodialysis patients," Kidney International, vol. 69, no. 9, pp. 1626-1632, 2006.

[13] R. L. Pisoni, B. Wikstrom, S. J. Elder et al., "Pruritus in haemodialysis patients: international results from the Dialysis Outcomes and Practice Patterns Study (DOPPS)," Nephrology Dialysis Transplantation, vol. 21, no. 12, pp. 3495-3505, 2006.

[14] N. Senturk, O. Ozkaya, S. Aytekin et al., "Characteristics of pruritus in children on peritoneal dialysis," Nephron. Clinical Practice, vol. 109, no. 3, pp. c168-c172, 2008.

[15] E. A. Attia, S. I. Hassan, and N. M. Youssef, "Cutaneous disorders in uremic patients on hemodialysis: an Egyptian casecontrolled study," International journal of dermatology., vol. 49, no. 9, pp. 1024-1030, 2010.

[16] E. Balaskas, J. C. Szepietowski, D. Bessis et al., "Randomized, double-blind study with glycerol and paraffin in uremic xerosis," Clinical Journal of the American Society of Nephrology, vol. 6, no. 4, pp. 748-752, 2011

[17] G. Yosipovitch, "Dry skin and impairment of barrier function associated with itch - new insights," International Journal of Cosmetic Science, vol. 26, no. 1, pp. 1-7, 2004.

[18] I. Zucker, G. Yosipovitch, M. David, U. Gafter, and G. Boner, "Prevalence and characterization of uremic pruritus in patients undergoing hemodialysis: uremic pruritus is still a major problem for patients with end- stage renal disease," Journal of the American Academy of Dermatology, vol. 49, no. 5, pp. 842-846, 2003.

[19] C. A. Morton, M. Lafferty, C. Hau, I. Henderson, M. Jones, and J. G. Lowe, "Pruritus and skin hydration during dialysis," Nephrology, Dialysis, Transplantation, vol. 11, no. 10, pp. 2031-2036, 1996.

[20] A. Markova, J. Lester, J. Wang, and L. Robinson-Bostom, "Diagnosis of common dermopathies in dialysis patients: a review and update," Seminars in Dialysis, vol. 25, no. 4, pp. 408-418, 2012.

[21] P. Aramwit and O. Supasyndh, "Uremic pruritus; its prevalence, pathophysiology and management," in Intech Open Sci, pp. 19-41, 2015.

[22] C. Filippi, R. Regazzini, V. Piazza et al., "Uraemic pruritus is not related to plasma histamine concentrations," Clinical and experimental dermatology., vol. 20, no. 4, pp. 294-296, 1995.

[23] V. Piazza, F. Cd, and C. Aprile, "Uraemic pruritus and plasma histamine concentrations," Nephrology Dialysis Transplantation, vol. 8, no. 7, pp. 670-671, 1993.

[24] A. Harlim, "Factors associated with pruritus uremic in chronic kidney failure patients," Journal of Pakistan Association of Dermatology, vol. 30, no. 1, pp. 86-97, 2020. 